\title{
Design and Validation of Deployable and Lockable Mechanism for Gossamer Spacecraft
}

\author{
SUN Liang ${ }^{1}$, Ji Ming ${ }^{1}$, Li Haizhi ${ }^{1}$, WANG Jin ${ }^{1}$, KANG Changxi ${ }^{1}$, YAN Bin ${ }^{1}$, YAO Lei ${ }^{1}$, KOU Zongyu ${ }^{1}$ \\ ${ }^{1}$ Lanzhou Institute of Physics, Lanzhou, 730000, China
}

\begin{abstract}
This passage introduces the researches of Deployable and Lockable mechanism for a new Gossamer Spacecraft built by space apply which can be locked and deployed by its own. This mechanism integrates deploying and locking. Its advantages are simple and reliable. It is based on a serious project "Platform Technology of Gossamer Spacecraft" from Lanzhou Institute of Physics. This project manufactured a model machine, finished the ground experiment, passed the project check. This passage introduces work theory particularly, builds the dynamics models, analyzes experiment results factors and presents measurement methods. Results show that the mechanism designed can meet the project requirement; it can build the foundation for the super-large Gossamer Spacecraft of China and improve the degree of the Aerospace technology application.
\end{abstract}

\section{INTRODUCTION}

As a new type of spacecraft platform, gossamer spacecraft has attracted wide attention at home and abroad. Pressure of sunlight is used to be a driving force to carry out space flight without fuel, and carry more space loads to achieve the goal of faster, cheaper and better. For on-orbit deep space exploration, gossamer spacecraft platform has a huge opportunity.

There are two basic types of gossamer spacecraft platform, one is non-skeleton, which uses rotating centrifugal expansion, and the other one is skeleton, which uses inflatable expansion. The non-skeleton is widely used in foreign countries, like the NASA's Jet Propulsion Laboratory and Langley Research and Development Center have developed a 20 meter demonstration prototype $^{[1,2]}$ and the European Space Agency has developed a biased reflector antenna, which is a support structure for inflatable telescope hood and a parachute of inflatable expansion. Inflatable research is studied in the United States and Russia ${ }^{[3,4]}$. At present, China Academy of Space Technology has developed a skeleton and inflatable 25 meter ground demonstration prototype and smooth inflatable work ${ }^{[5]}$.

The ground demonstration prototype which is developed by Lanzhou Institute of Physics is mainly composed by a deployable and lockable mechanism and a gossamer sails body. The aim of the deployable and lockable mechanism is to store gossamer sails body as much as possible in a limited space, to maintain a certain shape, and not to affect the inflatable process of gossamer sails. The deployable and lockable mechanisms can provide effective restraint and protection to the support arms and sails which are folded during the launch phase. Before the operation of the spacecraft, the mechanism is deployed to the designated position and locked to maintain the working state of the locking plane, waiting for the support arms and sails smooth to be deployed.

\section{Mechanism composition and design}

\subsection{Mechanism Research status}

The deployable and lockable mechanism is earliest used in the spacecraft mechanism organizations, and is most widely used in the spacecraft mechanism organizations. The deployable and lockable mechanism can be divided into three main types according to the principle of operation: strip semi-rigid, locking jaw type and connecting rod ${ }^{[6]}$.

The typical application of the strip semi-rigid deployable and lockable mechanism is to become a belt separation device which is used adapter connect and separate in satellite and rocket. This technology has become a mature technology, so China and other major space countries have mastered the technology.

The locking jaw type deployable and lockable mechanism is a more mature of lockable and released form in the world. This locking and releasing type is used in solar arrays and antennas. The typical examples are solar arrays lockable and released mechanism of the Intelsat-v satellite and the Astro-AeroSpace company has developed a lockable and released mechanism of AstroMesh flexible antenna. China has used this mechanism in the solar arrays of FY-1 satellite and 
antennas of ShenZhou spacecraft, as also, there is no relevant application in large and heavy weight object up to now.

The most typical applications of connecting rod deployable and lockable mechanism are the solar arrays and some of the connection separation bolts, and now China and other major countries have mastered this technology, and it is widely used to achieve the connection and separation between solar arrays and cabin in star ships and other spacecrafts.

\subsection{Mechanism composition}

Taking re-use and energy saving into account, hinge torsion-spring mechanism can be used in this project. Deploy function after releasing will be driven by preloaded torsion springs which have been installed in each hinge of product. According to light weight and small size constraints, the rotary hinge locking device can achieve a high locking force and locking stiffness, can easily carry out the ground unlock action to reset and test experiments, and also can achieve a higher locking repeatability.

In this paper, the deployable and lockable mechanism is used to lock and deploy membrane sail body of the spacecraft, before reaching the track. Its role is to maintain the lockable state, protect and restrain the sail body. After reaching the track, its role is to unlock, deploy and automatic lock. When it reaches the predetermined position angle, it ensures the opening board to be flat, provides a platform for the sail to inflate and deploy. The three-dimensional schematic diagram of the mechanism is shown in Fig1.

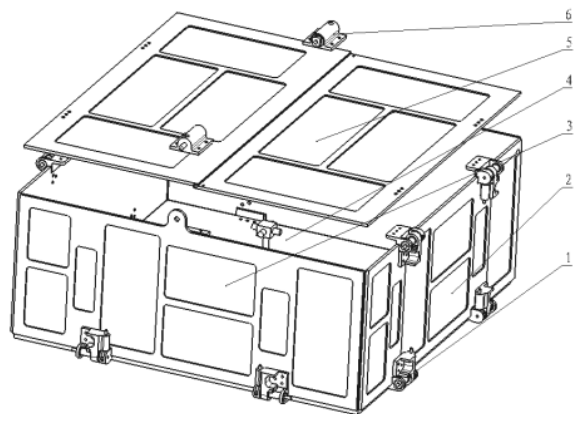

1- Rotary hinge 2- Side plate I 3- Side plate II 4- Base plate 5-Roof plate 6-Lockable and releasable mechanism

Figure.1 Deployable and Lockable Mechanism Model

The base plate serves as a platform for mounting other components. Side plate I has two plates, parallel to each other, side plate II has two plates, parallel to each other too, roof plate has two plates, located in the same plane. Side plate I and side plate II are fixedly attached to the base plate by means of a rotary hinges, roof plate is fixed attached to the side plate I by means of a rotary hinges. The lockable and releasable mechanism is mounted on the roof plate by screws, locking function is finished by compression spring pretension force and limit hole of side plate II, the pressure generated by the intake valve is used as the power source for the release of the mechanism. The torsion spring of rotary hinge is used as the power source for the deploying of the mechanism,

and the compression spring is used as the power source for the locking when it reached the scheduled position.

\subsection{Deployable and lockable mechanism}

The deploying and locking functions are integrated in the rotary hinge assembly. The rotary hinge assembly consists of male hinges, female hinges, rotary shafts, torsion springs, compression springs and locking pole. The three-dimensional schematic diagram of the rotary hinge is shown in Fig2a.

The torsion spring, rotary shaft, male hinge and female hinge complete the deploying function of the mechanism, torsion spring as energy storage component, the initial state is the state of force, the potential energy which is stored will transform into the kinetic energy to rotate the male hinge and the female hinge, the size of the output force can be changed by adjust the torsion spring installation position, the deploying function of mechanism is achieved. The compression spring, locking pole and slide rail are fully engaged in the locking function, the initial state of compression spring is in the working state, and the potential energy stored in the compression spring is converted into the kinetic energy to finish forward movement of the lock pole, when the locking pole is inserted into the locking hole, the locking function of mechanism is achieved. The schematic diagram of the locking function is shown in Fig2b.

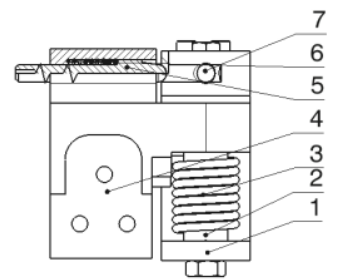

(a)Initial State (b) Lockable state

1- Male hinge 2- Rotary shaft 3- Torsion spring 4- Female hinge 5-Locking pole 6-Compression spring 7- Slide rail

Figure.2 Rotary Hinge Assembly Model

\subsection{Lockable and releasable mechanism}

The lockable and releasable mechanism is mounted on the roof plate. It consists of two pneumatic valves which can provide locking and release function for the roof plate of gossamer spacecraft.

The pneumatic valve form is used in lockable and releasable mechanism. It mainly contains piston shaft, cylinder, compression spring, end cap and O-shaped rubber ring etc. The three-dimensional schematic diagram of the rotary hinge is shown in Fig3. The working principle is as follows: before the spacecraft works, the roof plate shall is kept in the closed state. At this time, the motive force of the piston rod movement is provided by the deformation of the compression spring. When the piston rod is moved leftward into the limit hole, the pressing function is realized. After the work of the spacecraft, the intake valve through the hole of cylinder aerate into a certain pressure of the gas, pushing the piston rod to be moved by the opposite direction, to 
promote the compression spring compress and deformation, the piston rod will separate from the limit hole of the side plate, to achieve the release function of spacecraft. The schematic diagram of the locking function is shown in Fig4.
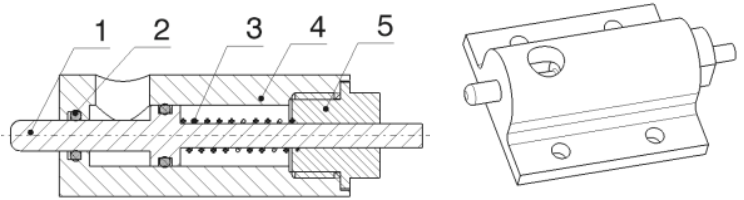

1- Piston shaft 2- O-shaped rubber ring 3- Compression spring 4-Cylinder 5End cap

Figure.3 Lockable and Releasable Mechanism Model

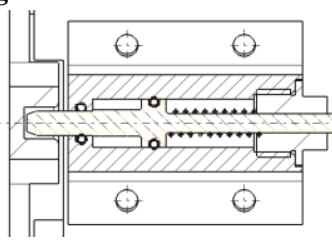

(a)Locking State

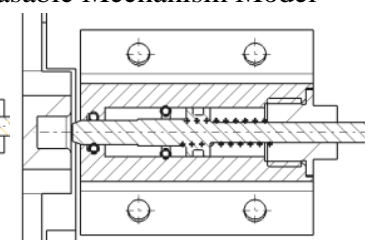

(b) Releasing State
Figure.4 Lockable and Releasable Mechanism Schematic

Diagram

\section{Dynamics models}

The dynamically modeling for the deployment process of deployable and lockable mechanism is built, and the deployment process will be simplified to that a hinge rotates around the axis. All the driving forces of the mechanism are derived from the torsion springs in the hinges and the drive torque of the torsion spring is related to the rotate angle. This article uses the deployable analysis method of the trusses, $3 n$ Cartesian coordinates are used as unknown vectors, and the relationship between unknown vectors and the angle of the torsion spring will be established. The kinetic equation of the torsion spring drive is deduced. Based on the first Lagrangian equation applied to the rigid body, the torsion spring drive has the elastic deformation energy ${ }^{[7]}$. The kinetic equation is:

$$
\sum_{i=1}^{3 n}\left(\frac{d}{d t} \frac{\delta T}{\delta \dot{x}_{i}}-\frac{\partial T}{\partial x_{i}}-\frac{\partial V}{\partial x_{i}}\right) \Delta x_{i}=(\Delta X)^{T}\left(\frac{d}{d t} \frac{\delta T}{\delta \dot{X}_{i}}-\frac{\partial T}{\partial X_{i}}-\frac{\partial V}{\partial X_{i}}\right)=0
$$

In the equation, $V$ is the kinetic energy of the system, $T$ is the elastic deformation energy of the system, and $X$ is the generalized coordinate array of the nodes.

In the process of deploying, the torsion spring drive force is also changing at times with the angle of change; the torque has the following formula equation:

$$
M=K\left(a_{0}-a\right)
$$

In the equation, $M$ is the driving torque; $K$ is the stiffness coefficient of torsion spring, is the initial twist angle of the torsion spring, and is the twist angle of torsion spring which is changing in the movement. The elastic deformation of torsion spring can be:

$$
V=1 / 2 K\left(\alpha_{0}-\alpha\right)^{2}
$$

Bring the elastic deformation formula equation into the equation (1); you can deduce the generalized driving force of torsion spring driving:

$$
F=\frac{\partial V}{\partial x}=K\left(\alpha_{0}-\alpha \frac{\partial \alpha}{\partial x}\right)
$$

After calculating the driving force of the torsion spring, all the torsion spring driving forces in the mechanism are assembled according to the finite element method, forming a generalized driving force array. And the generalized driving force array is different at the different time of the movement, which is changed with the change of the different node positions. At now, the dynamic equation of the torsion spring drive structure is:

$$
\sum_{i=1}^{3 n}\left(\frac{d}{d t} \frac{\delta T}{\delta \dot{x_{i}}}-\frac{\partial T}{\partial x_{i}}-\frac{\partial V}{\partial x_{i}}\right) \Delta x_{i}=(\Delta X)^{T}\left(\frac{d}{d t} \frac{\delta T}{\delta \dot{x_{i}}}-\frac{\partial T}{\partial x_{i}}-Q(x, t)=0\right.
$$

\section{Ground validation and factors}

Through above design process and dynamic models, as well as research and analysis a large number of domestic and foreign literatures, we have manufactured a demonstration prototype, conducted a ground experiment.

\subsection{Ground demonstration validation}

The aluminum alloy 2A12 is selected as the main material of the deployable and lockable mechanism, the stainless steel $1 \mathrm{Cr} 18 \mathrm{Ni}$ Ti is used as the main material of the rotating hinge assembly and the lockable and releasable mechanism, and the wire $65 \mathrm{Mn}$ serves as the material of the compression spring and the torsion spring. The physical dimension of the demonstration prototype is: $550 \mathrm{~mm} \times 550 \mathrm{~mm} \times 250 \mathrm{~mm}$, its weighs about $9.5 \mathrm{Kg}$. The real product of the deployable and lockable mechanism shown as Fig5:

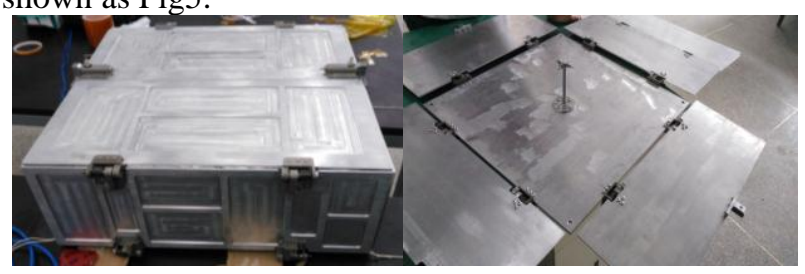

(a) Initial State of Product (b) Locking State of Product

Figure.5Product of Deployable and Lockable Mechanism

\subsection{Factors discussion}

From the results of the ground demonstration experiment, it can be found that the main factors affect the acceleration of the deployment process and the time of the deployment process: the friction of mechanism and spring stiffness of torsion spring.

(1) the impact of mechanical friction

In the ground experiment, the parts of the mechanism did not carry out lubrication treatment, astringent phenomenon had appeared in the deploy process, the deploying starts slowly, about 5 seconds, through the measurement and calculation, the friction between the side plate I and the base plate is about 2.6 Newton, The friction between the side plate II and the base plate is 
about 2.8 Newton.

After the grease was applied to the parts of the mechanism, the astringent phenomenon disappeared, the velocity was relatively increased, deploying time is about 3 seconds, the acceleration is also relatively large, but the smoothness of the deployment process was affected, the impact force is also large when it works, side plates appeared jitter phenomenon.

(2) the impact spring stiffness of torsion spring

In the ground experiment, astringent phenomenon appeared in the deployment process of roof plate, on one hand it is the impact of friction, and on the other hand it is due to the spring stiffness of the torsion spring. After measurement and analysis, the resistance moment of one single roof plate is about 100 Newton millimeter, the torsion spring stiffness coefficient $\mathrm{K}$ initial selected is 1.5 times margin, the torsion spring stiffness coefficient $\mathrm{K}$ rewinding is 2 times margin, the astringent phenomenon disappeared in the deployment process, the velocity was relatively increased, deploying time is about 3 seconds.

It can be seen that the friction of the mechanism and the stiffness of the torsion spring are the main influencing factors of the acceleration of the deployment process and the deployment time of mechanism. According to the requirements such as deployment time, deployment acceleration and the impact force, it is recommended to apply the lubricating or grease to the mechanism to reduce the friction, the torsion spring stiffness coefficient margin recommended 1.8-2.0.

\section{Conclusions}

1) For the new deployable and lockable mechanism, design is clever, set deployment and locking function as a whole, takes up a small size, its weight is light, simple and reliable.

2) The torsion spring-type device is used; convert potential energy into the kinetic energy by the use of energy conversion principle, function is achieved, without additional energy, which can be repeated several times in the ground experiment.

3) Manufacture a demonstration prototype, conduct a ground experiment, the deployment and locking of the mechanism are fast and reliable, and analyze the factors which affect the development process, and put forward the countermeasures and suggestions.

\section{References}

1. John Huang, Houfei Fang, Richard Lovick, Michael Lou. The Development of Large Flat in floatable Antenna for Deep-Space Communications [C]. AIAA, 2004.

2. Robert R. Romanofsky. Inflatable Membrane Reflector and Shape-Memory Polymer Antenna Developed for Space and Ground Communications Applications [C]. NASA, 2006.

3. Veldman S L, Ver meeren C AJr.Inflatable structures in aerospace engineering-an overview $[\mathrm{D}]$. Composite Material sand Structures Faculty of Aerospace Engineering, Delft university of technology , 2002.

4. Rohwelle D J. @qualification of the inflatable sunshield in space (ISIS) mast [C] . Proceeding of the 36t $\mathrm{h}$ Aerospace Mechanisms Symposium. Glenn Research Center, 2002.

5. David Cadogan, Craig Scheir, Anshu Dixit, and Jody Ware, Emily Cooper, Dr. Peter Kopf. Intelligent Flexible Materials for Deployable Space Structures (InFlex). AIAA 2006-1897.

6. Kriss J.Kennedy. Inflatable Habbitats Technology Development. NASA20050182969.

7. Juan R. Cruz, J. Stephen Lingard.Aerodynamic Decelerators for Planetary Exploration: Past, Present, and Future NASA20060028185. 